\title{
EDUCACIÓN FÍSICA DE CALIDAD: ¿DE DÓNDE SURGE ESTE PLANTEAMIENTO?
}

\section{Quality Physical Education: Where Does this Approach Come From?}

Rosa López-D’Amico'1

rlopezdedamico@yahoo.com

${ }^{1}$ Universidad Pedagógica Experimental
Libertador -Maracay, Venezuela

Fecha recepción: 9/7/2019

Fecha aprobación: 2/11/2019

\section{Resumen}

La Organización de las Naciones Unidas para la Educación, la Ciencia y la Cultura (UNESCO), ha generado en los últimos años orientaciones en torno a la Educación Física de Calidad (en adelante EFC), con el objeto de enfatizar en las naciones el valor de la práctica sistemática y su presencia en el currículo educativo. Las discusiones acerca de la EFC vienen desarrollándose desde hace más de 15 años. En el reporte de la UNESCO (2005) acerca de la calidad de la Educación Física se destacó la importancia de áreas esenciales, tales como la eficiencia del desarrollo de un estilo de vida activo, aspectos de salud, la calidad del crecimiento estudiantil en aspectos relacionados con valores y actitudes hacia el deporte y la actividad física, hábito de la práctica regular de ejercicios y preocupación acerca de su eficiencia para enfrentar los retos como los temas de género, educación inclusiva, aspectos raciales y limitaciones religiosas, tradicionales o culturales. El proceso del desarrollo con calidad no está restringido al desarrollo curricular o métodos de formación, también se relaciona con la construcción del ambiente, políticas, formación y desarrollo del personal. Es decir, involucra un estado holístico de características esenciales que permita a la Educación Física alcanzar su libertad en el accionar. En este trabajo, se presenta una revisión de manera descriptiva de lo que se ha venido produciendo a partir de la UNESCO en materia de la EFC y su aplicación en algunas naciones.

Palabras clave: actividad física, Educación Física, calidad, actitudes, valores.

\section{Abstract}

In recent years, the United Nations Educational, Scientific and Cultural Organization (UNESCO) has generated guidelines towards Quality Physical Education (QPE) in order to emphasize the value of systematic practice in nations and its presence in the educational curriculum. Discussions about QPE have been going on for more than 15 years. UNESCO's report (2005) on the quality of physical education highlighted the importance of essential areas, such as the efficiency of the development of an active lifestyle, health aspects, the quality of student growth in aspects related to values and attitudes towards sport and physical activity, habit of regular exercise practice and concern about their efficiency to address challenges such as gender issues, inclusive education, racial issues and religious, traditional or cultural limitations. The process of quality development is not restricted to curriculum development or training methods, it also relates to the construction of the environment, policies, training and development of staff. That is, it involves a holistic state of essential characteristics that allows physical education to achieve its freedom in action. This work presents a descriptive review of what has been produced from UNESCO on the field of QPE and its implementation in some nations.

Keywords: attitudes, quality, Physical Education, physical activity, values. 


\section{Introducción}

Este trabajo presenta un recuento de carácter histórico-teórico del planteamiento de la Educación Física de Calidad (EFC) que se ha venido impulsando desde la UNESCO. Está basado en experiencias propias de la autora quien ha participado en algunas fases de este desarrollo histórico en los últimos años como miembro de organizaciones, investigadora y revisora de políticas de la EFC. El artículo está estructurado en ocho partes, se inicia con esta introducción para explicar la organización de la información, luego con la sección denominada situación, contextualización que aborda brevemente la evolución de la Educación Física como elemento de la sociedad y del sistema educativo. Posteriormente, se clarifican las organizaciones que son los referentes para la agenda de la Educación Física, Deporte y Actividad Física a nivel global, y que son para esta área lo que la Organización Mundial de la Salud es para el sector salud o la UNESCO para la educación. En la siguiente sección, de manera sucinta, se define el término Calidad y su utilización en la UNESCO. Luego, se encuentra la sección del desarrollo de la temática EFC y su evolución en los últimos 20 años, y continúa con el apartado que recopila la respuesta a nivel mundial por parte de la UNESCO y diversas organizaciones para valorar, hacer un llamado a las naciones y resaltar la importancia de la Educación Física y el Deporte como un derecho humano y su sitial en el sistema educativo. Por último, se hacen los comentarios finales con sugerencias para Latinoamérica y se cierra con las referencias.

\section{Situación}

La práctica variada de la Educación Física ha sido siempre atractiva en el sistema educativo. Algunos estudiantes reciben la clase de Educación Física con una calidad excelente, aunque el concepto de excelente podría variar, en este caso se refiere a tener un docente bien preparado con una visión de una educación holística que practique el área del conocimiento desde la interdisciplinariedad y que busque el desarrollo biopsicosocial del estudiante. Si bien es cierto que conseguir el perfil anterior no es tarea fá- cil, existen además a nivel global restricciones para la práctica de una Educación Física inclusiva, limitaciones provocadas por la cultura, religión, economía y status social, entre otros aspectos. Incluso, algunos nunca han recibido una clase de Educación Física.

Para abordar la temática de este artículo, es necesario referir la relevancia del deporte en la sociedad y su evolución en el sistema educativo. El deporte ha existido desde los primeros estadios de la sociedad humana de manera diversa, por ejemplo, como destrezas básicas para la sobrevivencia como la caza, la lucha y la guerra. Las civilizaciones antiguas como la china, egipcia, india, azteca, maya, etc., dejaron muestras de esas experiencias y el deporte les sirvió como parte de la vida en competencias, tácticas militares de entrenamiento y artes marciales. La necesidad de prepararse para la sobrevivencia permitió a los seres humanos desarrollar los componentes físicos, sociales, emocionales y psicológicos. Estos componentes involucran el desarrollo de nervios, músculos, competencia física y otras cualidades.

Amusa y Toriola (2010) señalan que en la mayoría de países y comunidades africanas, la cultura física forma una parte integral de los procesos tradicionales asociados con la cacería, actividades pastorales, alimentación, conflictos inter-tribales, sobrevivencia y buena salud. Esto se evidencia en numerosos juegos indígenas, ritos de iniciación y otros rituales. Las tribus Masái de África Oriental, Los Zulúes de Sudáfrica, Los Yorubas de la sub-región de África Occidental, Los Tuaregs y árabes beduinos de África del Norte, entre otras, desarrollaron juegos tradicionales, deportes, actos, bailes y artes que fueron usados para varios propósitos de socialización, iniciación, ceremonias, recreación, etc.

El deporte emergió de los ejercicios y los atletas lo usaban para preparar sus cuerpos y mentes para la guerra, y esto jugó un rol muy importante en el desarrollo del deporte hacia una forma de cultura popular y elemento de la sociedad. Por ejemplo, competencia de caminata, boxeo, lucha y carreras de carretas. El trabajo de la Educación Física indicaba el deseo de organizar las actividades y técnicas de combate con 
procedimientos de entrenamiento. El propósito de los entrenamientos era ayudar a los atletas a madurar las destrezas para estar listos para la competencia. El énfasis en anatomía, habilidades y logros físicos sirvieron como elementos esenciales en la vida de las personas, y el sistema de las escuelas atenienses estaba basado en el entrenamiento físico, la música y las actividades deportivas. Esta forma de educación se reflejaba en la tradición de deporte y competencia en los Juegos Olímpicos en el periodo 776 a. C. al 339 d.C. (Demirel \& Yildiran, 2013).

Los intentos iniciales de la educación del movimiento en las escuelas se desarrollaron a partir de actividades gimnásticas y ejercicios físicos. El desarrollo de la Educación Física moderna y el movimiento gimnástico en la educación se presenta con Johann Bernhard Basedow (1723-1790), quien fue el primero en reconocer la importancia del ejercicio; requería a sus estudiantes un uniforme específico que les permitiera la libertad irrestricta del movimiento y ofrecía un campamento para niños por dos meses durante el verano. El padre de la Educación Física moderna, como la conocemos hoy en día, es Friedrich Ludwig Jahn (1778-1852), quien estableció el primer gimnasio para niños en Alemania. Creía que la mejor sociedad era aquella que había establecido los estándares para la fortaleza física y las habilidades. En 1813, el maestro suizo Pehr Henrik Ling (1776-1839) tuvo éxito en desarrollar el Instituto Gimnástico Central Real, con la cooperación del gobierno suizo, el cual logró avanzar en el acondicionamiento físico (Ho, 2018).

El movimiento gimnástico se hizo más común y contribuyó al desarrollo de las actividades gimnásticas al aire libre en 1800. El primer Turnplatz, o gimnasio al aire libre fue abierto por Jahn en Berlin en 1811, y el trabajo de Turnverein (asociación de gimnasia) se extendió rápidamente (Goodbody, 1982). Programas deportivos extraescolares se implementaron en escuelas públicas en Inglaterra con el auge de las ciencias naturales y especialmente la Biología. El desarrollo muscular se enfatizó con el eslogan "para educar la mente hay que educar el cuerpo", entonces, la fascinación por la salud conllevó la obsesión por la práctica del deporte y el juego, principalmente reflejada en las escuelas públicas para niños en Inglaterra. El atleta se convirtió en un héroe de la sociedad en la época victoriana (Galakof, 2015).

El desarrollo de los programas de Educación Física en los Estados Unidos de América tiene como referente a Charles Beck (1798-1866), quien fue amigo y seguidor de Jahn. Beck fue contratado para enseñar Latín y Educación Física en la forma de Gimnasia Alemana, convirtiéndose en el primer docente oficial de Educación Física en los Estados Unidos de América. Diocletian (Dio) Lewis (1823-1886), hizo más para la promoción de la Educación Física que cualquier otra persona. Quiso que los débiles, mayores, gordos, frágiles y mujeres tuvieran un sistema que pudieran usar. Abrió el Instituto Normal de Educación Física en Boston, inventó bolsas de frijoles, pesas de madera y también usó música para exaltar sus ejercicios.

El desarrollo de la Educación Física en el siglo XX es planteado por muchos autores, así como las diferentes tendencias y enfoques, por ejemplo, Ramírez (2009) y López de D’Amico (2013). No obstante, a pesar de la larga presencia de la Educación Física en el sistema educativo, Hardman y Marshall (2000) levantaron las alarmas acerca de la pobre situación en que se encontraba la Educación Física en los sistemas educativos, a través de un estudio de carácter global, que incluso tuvo su seguimiento y posteriores publicaciones. A partir de ese entonces se desarrolló una serie de actividades en pro de elevar el estatus de la Educación Física.

\section{Organizaciones de toma de decisiones}

En 1976 se realiza por vez primera la Conferencia Internacional de Ministros y Altos Funcionarios Encargados de la Educación Física y el Deporte (MINEPS), foro que facilita el intercambio de conocimientos técnicos e intelectuales en materia de Educación Física y Deporte. El MINEPS también sirve como mecanismo institucional para una estrategia internacional coherente en este ámbito. Asimismo, este foro tuvo un 
papel importante en el desarrollo de la Carta Internacional de la Educación Física y el Deporte (1978) — estableciendo la práctica de la Educación Física y el Deporte como un derecho fundamental para todosy en la creación del Comité Intergubernamental para la Educación Física y el Deporte (CIGEPS). También provee una plataforma clave para la negociación de la Convención Internacional contra el Dopaje en el Deporte. Hasta la fecha se han realizado seis MINEPS, a saber: París (Francia), 1976; Moscú (URSS), 1988; Punta del Este (Uruguay), 1999; Atenas (Grecia), 2004; Berlín (Alemania), 2013 y Kazán (Federación de Rusia), 2017. Entre congresos se han realizado reuniones de seguimiento a las decisiones, como por ejemplo, la realizada este año en Madagascar (región África). De cada MINEPS se han generado sendos informes y allí se han tomado las decisiones en cuanto a las orientaciones hacia la Educación Física, el Deporte y la actividad física.

El Comité Intergubernamental para la Educación Física y el Deporte (CIGEPS) fue establecido en 1978 para desarrollar el papel y el valor del deporte, así como para promover su inclusión en políticas públicas. El CIGEPS está compuesto por representantes de 18 Estados miembros de la UNESCO, expertos de la Educación Física y del Deporte, elegidos para un mandato de cuatro ańos. Cuenta además con un Consejo Consultivo Permanente (PCC), constituido por representantes de federaciones deportivas, agencias de las Naciones Unidas y organizaciones académicas reconocidas (ONG) para suministrar apoyo y sugerencias al Comité (UNESCO/CIGEPS, 2012). Para el ańo 2019, el CIGEPS cuenta con los siguientes Estados miembros de la UNESCO: Alemania, Argentina, Azerbaiyán, Bahréin, Colombia, Emiratos Árabes Unidos, Finlandia, Gambia, Japón, Madagascar, Malasia, México, Rusia, Suráfrica, Túnez, Turkmenistán, Turquía y Ucrania. El comité ejecutivo es electo cada dos años y actualmente está conformado por el ministro de Deporte de la República de Suráfrica como presidente, y cinco vicepresidentes que responden a las regiones geográficas establecidas: Alemania por el grupo I, Rusia por el grupo II, Colombia por el grupo III, Japón por el grupo IV y Túnez por el grupo V.
El PCC está conformado por: 1. Asociación General de Federaciones Internacionales de Deporte (GAISF); 2. Comité Internacional de Ciencias del Deporte y la Educación Física (ICSSPE); 3. Comité Olímpico Internacional (IOC); 4. Comité Paralímpico Internacional (IPC); 5. Fondo de las Naciones Unidas para los Niños (UNICEF); 6. Programa de las Naciones Unidas para el Desarrollo (UNDP); 7. Programa del Medio Ambiente de las Naciones Unidas (UNEP); 8. Oficina del Pacto Mundial de las Naciones Unidas; 9. ONU Mujeres; 10. Organización Mundial para la Salud (WHO, por sus siglas en inglés). Además, hay 21 organizaciones más que tienen membresía renovable. En este artículo se refiere en varias oportunidades a ICSSPE en virtud de que ha sido la organización académica que mayor seguimiento ha dado al tema de la EFC.

El Consejo Internacional de Ciencias del Deporte y la Educación Física (ICSSPE) fue creado en 1950. Es la organización académica de carácter internacional que agrupa organizaciones de carácter local, regional, nacional, continental e internacional de características científicas, académicas, deportivas y sin fines de lucro en general, que trabajan en el área de la Educación Física, Deporte, Recreación y Danza. Agrupa organizaciones tan variadas desde el nivel macro, por ejemplo, el Comité Paralímpico Internacional, científicas; la Federación Internacional de Medicina del Deporte, deportivas; Federación Internacional de Natación, etc. Tiene más de 300 organizaciones afiliadas. Anteriormente constituyó, en conjunto con otras organizaciones los congresos preolímpicos y en el 2013 y 2017 fue la organización académica que apoyó el desarrollo de MINEPS por medio de sus organizaciones miembros y sus expertos.

\section{Acerca de la calidad}

La UNESCO ha venido planteando desde hace tiempo el tema de la calidad en educación (UNESCO, 2004). En junio del 2000, en la ciudad de Florencia (Italia), este organismo produjo un papel de trabajo para definir calidad en educación, en una reunión de un grupo de trabajo en educación. El término "calidad" proviene del latín qualitas o qua- 
litatis. La percepción de la calidad puede ser individual y puede variar dependiendo de las expectativas de las personas o comunidad. Calidad es el grado en el cual un producto logra los requerimientos o demandas del cliente; la calidad tiene unas dimensiones básicas a conocer: técnica, humana y económica. El muy conocido el sistema ISO 9001 que no provee de calidad, no está diseñado para crear calidad, es un espacio (entidad) que regula por medio de unos estándares la calidad de los procesos. Entonces la calidad es también la experiencia del cliente, la percepción de la calidad del producto proviene de las especificaciones de diseño y los estándares de manufactura logrados.

El reporte de la UNESCO acerca de la Educación Física de Calidad fue producto del seminario de Porto Novo (Benín) en mayo de 2005, en una la reunión de expertos internacionales, jefes de organizaciones donde se forman en Educación Física y representantes gubernamentales para definir y estructurar una agenda en cuanto a la EFC. El reporte de la UNESCO (2005), presentó una estructura con elementos de referencia (estrategias) para considerar la Educación Física y el Deporte de calidad en el sistema educativo. Estos estaban orientados con los ítems siguientes: 1) Definición de Educación Física y Deporte (EFD) de Calidad y sus alcances; 2) EFD estructura o sistema de profesionalización/formación; 3) Formación del docente en cuanto al currículo y su acompañamiento en el ejercicio; 4) Investigación; 5) Sustentabilidad; Ambiente/ Infraestructuras y otros factores sociales que intervienen.

Se especificó, además, que los requerimientos del Deporte y la Educación Física en la escuela son:

- Objetivos del currículo en la EF: deporte en función objetivos educacionales.

- Adopción de criterios amplios para adquirir habilidades, a través del deporte, para la vida.

- Enfoque en la salud y la regularidad en la práctica deportiva y actividad física.

También se acordó en esa sesión de Porto Novo un plan de acción internacional para generar las refe- rencias acerca de la EFD de Calidad (2005-2015). Estas comprendían las siguientes acciones: 1) 2006 - 2o seminario internacional acerca de la EFD de calidad en el sistema educativo; 2) 2006 - Seminario internacional acerca de las estructuras de formación de docentes para EFD en los países de Asia, Arabia y Europa oriental; 3) 2006/2007- Redes regionales de expertos de EFD; 4) 2006/2007 Firma de memorandos de entendimiento entre la UNESCO y organizaciones regionales e internacionales relevantes; 5) 2006/2008- Primera fase de la universalización; 6) 2006/2015 Trabajo in situ con la EFD (UNESCO, 2005).

\section{Desarrollo de la temática en los últimos veinte años}

En la Primera Cumbre Mundial acerca de la Educación Física (1999) se acordaron los siguientes aspectos:

- La necesidad de unificar acciones a nivel mundial para mejorar la situación de la Educación Física para todos los niños.

- ICSSPE, se comprometió en monitorear el progreso y efectividad.

- Ejemplos de buenas prácticas y los indiscutibles efectos de la Educación Física de calidad, en el futuro las acciones coordinadas pueden mejorar la calidad de vida de los nińos a nivel mundial

En la Segunda Cumbre Mundial acerca de la Educación Física en el 2005 se destacan los siguientes aspectos:

- Desarrollo de estándares de calidad y puntos de referencia para la Educación Física - evidencia científica y contribución particularmente en el desarrollo personal y social.

- Desarrollo de estrategias modulares efectivas para asegurar el desarrollo futuro de la Educación Física como un componente esencial de la educación. 
- Integración de políticas de Educación Física de alta calidad y culturalmente sensibles a nivel mundial en la educación y las políticas deportivas.

En su reunión del comité ejecutivo en el año 2009, la ICSSPE reforzó sus grandes áreas de trabajo: Educación Física y Deporte de Calidad; hábitos a lo largo de la vida de deporte o actividad física; aspectos éticos en el deporte, y desarrollo general de la salud a través del deporte y la actividad física. Para ello, enfatizó en algunas estrategias para lograr el desarrollo de las áreas propuestas: motivar la discusión e investigación, realización de seminarios y talleres por parte de las asociaciones miembros en sus conferencias anuales; organización de talleres, seminarios y otras actividades académicas con el auspicio de ICSSPE; encuesta para monitorear el desarrollo al finalizar el año. También se programó trabajar en las regiones de modo colaborativo entre los coordinadores de las regiones. Por ejemplo: desarrollo de planes regionales, desarrollo de investigaciones pequeńas, pero efectivas y proyectos para el desarrollo, en particular, en países en desarrollo, organización de equipos de trabajo multidisciplinarios, publicaciones y reportes en conferencias regionales. Este trabajo estuvo acompañado por el grupo denominado Comité Internacional de Pedagogía del Deporte (ICSP), a través de las organizaciones académicas miembros, revisión de sus propuestas y reportes, el proyecto de ICSP de los requerimientos para la Educación Física (2009) y la Educación Física y Deporte de Calidad (2010). Estos generaron múltiples investigaciones (Ho et al., 2014, 2016, 2017, 2018; López de D’Amico et al., 2018).

A pesar de las propuestas, los informes de Ken Hardman señalaban que existía todavía debilidad en algunos países para la ubicación de la Educación Física en sus sistemas educativos, pobre implementación de políticas para lograr la EFD de calidad (Hardman, 2007, 2008; Hardman \& Marshall, 2009). Algunas organizaciones también señalaron que no era apropiado hablar de estándar sino de puntos de referencia o referentes de la EFD de calidad. Según Hardman (2008), había una estructura y un marco de referen- cia sólido y concreto que fue desarrollado para proveer recursos e información para el desarrollo de un programa de calidad para la Educación Física, pero había mensajes mixtos. Había evidencia de compromiso a nivel nacional con la Educación Física, pero un proceso lento para transformar esa iniciativa con acciones completas y concretas para la implementación de un plan. Nuevos pensamientos emergieron en Educación Física, "marginalización” fue el término más sonado para indicar la dificultad o el progreso insatisfactorio que existía en general. Entonces parecía que la pregunta era: ¿qué está mal? Para la cual se necesitaba respuesta si el desarrollo de la EFC se observaba como una forma de mejorar la calidad del aprendizaje de los estudiantes.

En África, Amusa y Toriola (2010) hacían una fuerte crítica acerca de los programas de EF y señalaban que no estaban presentes elementos básicos de las diversas culturas africanas. Asimismo, afirmaban que, si se deseaba que los programas de EF y Deporte tuviesen éxito en África, debían ser desarrollados, diseñados y formados para el continente africano. Debido a la diversidad cultural del continente, no era práctico importar modelos o programas del extranjero. Los modelos importados fueron diseñados para servir a situaciones específicas de los poderes coloniales pasados.

En el 2014, el Banco Mundial reportó el desarrollo educacional en el sur de Asia e indicó acerca de la necesidad de mejorar la calidad de la educación. El reporte señalaba el hecho de que las escuelas primarias estaban casi completamente financiadas, pero que la falta de eficiencia adecuada era una preocupación. Había urgencia en hacer más para mejorar la calidad de la educación en ese informe del Banco Mundial (Ho et al., 2016).

Con el pasar del tiempo, otros nuevos elementos han emergido, por ejemplo, en el proyecto en Singapur se enfatiza en aspectos puntuales para responder a necesidades de la población: motivar a los estudiantes a ejercitarse y lograr un estilo de vida saludable; transformación en la EF por medio de la enseñanza a través del juego; pruebas de condiciones físicas; 
orientación a los estudiantes con sobrepeso; dieta y premio a las escuelas que tengan bajo nivel de obesidad.

En Latinoamérica, en diversos estudios, se evidencia poco cumplimiento de las políticas para la práctica de la Educación Física en el sistema educativo, la necesidad de mayor formación de docentes y la mejora de la infraestructura deportiva (López D'Amico et al., 2014c; Ho et al., 2018). También, en una edición especial de la revista Actividad Física y Ciencias, publicada en el 2005 y dedicada a la Educación Física en Latinoamérica, hay planteamientos de Argentina, Brasil, Chile, Colombia, Costa Rica, Cuba, México y Venezuela. López de D’Amico et al. (2014b) señalaron que el problema para el desarrollo de la Educación Física en Venezuela ha sido que no ha contribuido a la creación de hábitos para la participación en actividades físicas para la vida y/o actividades recreativas. Este problema aumentó con la falta de docentes formados para trabajar la Educación Física con nińos y jóvenes en el sistema educativo, pues por muchos ańos estuvo enfocado en la técnica deportiva y no en una clase de Educación Física.

Una situación similar se señala en México, donde "muchas escuelas no tenían docentes de Educación Física en la clase” (López-Taylor et al., 2014, p.315). En otros casos, el problema es debido a que los directores de las escuelas no creen en el programa de Educación Física, o en los docentes, de quienes no tienen buena percepción, y no creen que la Educación Física contribuya en la formación (Ho et al., 2016).

\subsection{Preocupación en el mundo por la calidad de la educación y respuesta gubernamental}

China adoptó la terminología de Educación de Calidad (sù zhi jiào yù) (素質教育) como su tema central en la reforma educativa en la década del 2000, con el objeto de responder al enfoque que existía en cuanto a evaluación en la educación (Ho, 2018). En la ciudad de Hong Kong (RAE) el reporte de la Comisión de Educación en 1997 se enfocó en el desarrollo de la calidad de la educación escolar, esa fue la agenda central y este concepto se esperaba que se implementara en varias fases. Cambios curriculares se realizaron en algunos países. Por ejemplo, el Consejo para el Desarrollo del Curriculum (Hong Kong) produjo el material Physical Education Key Learning, guía curricular para el área. La Directiva de Educación del estado de Michigan generó la política acerca de la EFC en el 2003. El Ministerio de Educación de Canadá creó en el 2010 la guía curricular de Ontario para los grados desde el uno hasta el ocho, intitulado Health and Physical Education (Ho et al., 2018).

En su reporte del 2013, la Comisión Europea presentó evidencias acerca de la Educación Física y el Deporte en las escuelas en Europa. Por ejemplo, docentes en Letonia, Austria, Eslovenia y Finlandia fueron motivados para incluir actividad física en varias materias escolares, en lugar de limitarlo a una sola clase. En Alemania, Portugal y el Reino Unido se adoptó el enfoque cross-curricular para brindar a los estudiantes una perspectiva más amplia de los deportes y el ejercicio. La República Checa, Alemania y Noruega les enseñan a sus estudiantes las normas de tránsito para los peatones y ciclistas como parte del currículo de Educación Física. En Grecia, la República Checa y Polonia, la Educación Física ha enfatizado la importancia de familiarizar a los jóvenes con los ideales olímpicos y sus símbolos (Ho et al., 2016).

En los Estados Unidos de América también se hicieron esfuerzos desde la Asociación Nacional de Deporte y Educación Física (NASPE) y el Centro para la Prevención y Control de las Enfermedades (CDC), por ejemplo, con acciones tales como los estándares nacionales para Educación Física (NASPE, 2004), la identificación de parte de Le Masurier y Corbin (2006) de las diez razones más importantes para apoyar la implementación de los estándares NASPE en 2006 y las estrategias del CDC para mejorar la EFC en 2010. Por ejemplo, NASPE identificó algunas áreas de importancia: curriculum, instrucción, valoración, tiempo académico, la mejoría de aspectos como instalaciones, recursos y educación profesional. Todos criterios esenciales para el desarrollo de programas de calidad en Educación Física (NASPE, 2007). 
En Latinoamérica hay referentes importantes acerca de la EFD de calidad en Cuba, los cuales son muy reconocidos (Díaz et al., 2013, 2016). También hay referencias de cambios que se han implementado en los sistemas educativos para incrementar las horas de Educación Física en el sistema educativo, por ejemplo, en Educación Secundaria (liceo), y la transformación curricular en ese nivel en atención a los parámetros de la EFC a los objetivos sustentables del milenio, como en Venezuela (López de D’Amico \& Guerrero de Hojas, 2018).

Nuevos conceptos para el aprendizaje en Educación Física se comenzaron a presentar en diversos ámbitos. Instrucción innovadora: enseñanza por medio de los juegos para la comprensión; aprendizaje por medio de juegos; modelo de enseñanza reflexiva; enfoque centrado en el estudiante. Oportunidades de Experiencias de Aprendizaje: programa de actividades co-curriculares; actividades recreativas escolares; actividades extracurriculares; programas de deporte después de la escuela; deporte de fines de semana.

Ha existido respuesta del mundo para el desarrollo de la Educación Física en el sistema educativo, sería mezquino no mencionar los esfuerzos desde los años setenta del siglo XX, por ejemplo, los que viene desarrollando la UNESCO:

1978 - Carta Internacional de la Educación Física y el Deporte.

2003 - Declaratoria y Recomendaciones de UN Magglingen.

2005 - Reporte acerca de la Educación Física de Calidad.

2005 - Año Internacional del Deporte y la Educación Física.

2010 - Argumentos de la ICSSPE acerca de la Educación Física (ICSSPE).

2010 - Indicadores Internacionales para el sistema de la Educación Física (ICSSPE).

2013 - V Conferencia Internacional de Ministros y Representantes de Gobierno responsables de la
Educación Física y Deporte (MINEPS V).

2013 - Declaración de Berlín.

2015-La Carta Internacional de la Educación Física, la actividad Física y el Deporte. UNESCO 2015.

2015 Educación Física de Calidad - Guía para los representantes políticos.

2015 Educación Física de Calidad - Guía para los representantes políticos - Metodología.

2015 Proyecto de Políticas para una EFC - Proyectos piloto.

Hay varias organizaciones que han participado en proyectos de apoyo: ISCPES, IAPESGW, FIEP, IFAPA (López de D’Amico et al., 2014a). También se menciona a TAFISA y el proyecto Designed to Move, una llamada para repensar los diferentes enfoques en el desarrollo de la Educación Física de Calidad, construyendo así un ambiente con características positivas para fomentar el desarrollo de una vida activa: Ciudades Activas, Día Mundial de la Caminata, Día del Reto para la Salud, Trabajos Experimentales; Proyectos de Semillas. Todos ellos son intentos para lograr un concepto holístico para el desarrollo de una Educación Física de Calidad.

ICSSPE, particularmente su grupo de trabajo Comité Internacional de Pedagogía del Deporte, propuso su agenda Declaratoria de Posicionamiento de la Educación Física y los Aspectos Referenciales de la Educación Física. Estos pronunciamientos marcaron la introducción de un concepto holístico para entender el desarrollo de una Educación Física de Calidad.

En MINEPS IV, 2004, se planteó apoyo al desarrollo holístico de la Educación Física y el deporte de manera que se convirtieran en un elemento importante en los sistemas educativos y contribuyan en las reformas educativas con el objeto de que se tome más en cuenta la Educación Física y el deporte en el currículo escolar. En MINEPS V, 2013, se acordaron las siguientes líneas de trabajo: acceso al deporte como un derecho fundamental para todos, promocionar inversión en programas de Educación 
Física y deporte, preservar la integridad del deporte y orientaciones para la Educación Física de Calidad. Los principales temas abordados se refieren al acceso a la Educación Física y el deporte y la participación en ellos como un derecho, independientemente del género, el origen étnico, la edad, la discapacidad, la procedencia socioeconómica y la orientación sexual. El aspecto central fue la elaboración de estrategias y mecanismos para mejorar el acceso de las mujeres y las niñas, así como las personas con discapacidades. Un aspecto importante a destacar, es que una inversión sostenida en EFC no es una opción de política, sino un componente fundamental de la filosofía de todos los países en materia de deporte, y que las asignaciones presupuestarias no deben desviarse de la oferta pública de programas de Educación Física (UNESCO/MINEPS V, 2013).

MINEPS VI, se centró en tres temas que corresponden a las tres esferas principales en materia de políticas del Plan de Acción de Kazán: 1) Elaborar una visión integradora del acceso inclusivo de todos al deporte, la Educación Física y la actividad física. 2) Potenciar al máximo la contribución del deporte al desarrollo sostenible y la paz. 3) Proteger la integridad del deporte.

La EFC se refuerza como una prioridad en dos áreas de políticas principales del Plan de Acción de Kazán, que fue aprobado en MINEPS VI: I.3: 1. Impulsar la EFC y las escuelas activas. 2. Brindar una educación de calidad y promover el aprendizaje para todos y la adquisición de competencias a través del deporte.

La Carta Internacional de la Educación Física, la actividad física y el Deporte, es un documento inspirado en los DD: HH. que fue adoptado por los Estados miembros de la Organización de las Naciones Unidas para la Educación, la Ciencia y la Cultura (UNESCO), el 18 de noviembre del 2015, durante la 38 a sesión de la Conferencia General de la UNESCO. Este documento es el legítimo sucesor de la Carta Internacional de la Educación Física y el Deporte, adoptada originalmente en 1978, durante la 20a Conferencia General de la UNESCO. La carta original, enmendada en 1991, fue el primer docu- mento inspirado en los DD. HH. en declarar que la práctica de la Educación Física y del deporte es un derecho fundamental de todos. Contiene 12 artículos que sirven como referente universal en materia de estándares éticos y cualitativos de la Educación Física, la actividad física y el deporte. Valores presentes en la Carta: el acceso al deporte es un derecho fundamental de todos, valores y beneficios del deporte, los roles de los diferentes actores, los principios de calidad y de ética.

La Carta es un gran documento que ampara y protege, en la que se visualiza la amplitud del área del conocimiento que implica la Educación Física. En el 2005, en el Reporte acerca de la Educación Física de Calidad, se resumía la discusión en cuanto al tema, extendiéndolo hacia otras áreas esenciales, como el desarrollo de un estilo de vida activo eficiente, aspectos de la salud, el crecimiento del estudiante en valores y actitudes en el deporte y la actividad física. Según la UNESCO (2015c), la EFC es un elemento central inicial para aprender hábitos de vida y desarrollar patrones de comportamiento positivos. La EFC es un punto de partida esencial para que los niños aprendan competencias para la vida y desarrollen modelos positivos de comportamiento.

Desde la UNESCO, las últimas acciones por la EFC comprenden los estudios pilotos que se vienen realizando en cinco países (Fiyi, México, Sudáfrica, Túnez y Zambia) con el Proyecto de Políticas de EFC (UNESCO, 2015c). La producción de los documentos Educación Física de Calidad - Guía para los representantes politicos (UNESCO, 2015a) y Educación Fisica de Calidad - Guia para los representantes politicos - Metodología (UNESCO, 2015b) ha impulsado para que los que trabajan con las políticas puedan tener unas orientaciones acerca de este desarrollo. Este proyecto y la construcción de los materiales citados, incluida una página web, ha sido una iniciativa de UNESCO, conjuntamente con otras organizaciones, a saber, IOC, la Comisión Europea, ICCSPE, Nike, la WHO, el PNUD y UNICEF para coadyuvar en este movimiento global de la EFC y que los países tengan una estrategia para su desarrollo, pues en las reuniones de MINEPS muchos los han solicitado. 
En la página web de la UNESCO puede encontrarse información en varios idiomas y también hay avances de los reportes presentados del desarrollo de este proyecto en Fiyi, México, Suráfrica y Zambia (UNESCO, 2017). También en estos países se han producido sendos documentos analizando el estado del arte y las perspectivas. Por ejemplo, en México, Ceballos et al., (2018). La idea principal es ver la política de la EFC y la puesta en práctica de la misma. Hasta ahora las políticas revisadas promueven la alfabetización física y el aprendizaje basado en valores, formando parte de un desarrollo completo y de las prioridades mundiales en materia de educación, pero todavía hay que hacer su implementación para ver si se logra la EFC con inclusión. Los países que participan deben expresar su interés, pero además el proceso de revisión prioriza el compromiso de las partes interesadas involucradas y afectadas por la política de EFC, a través de consultas diseñadas para reflejar las perspectivas en el terreno en los marcos normativos en donde participan evaluadores internos y externos.

\section{Comentarios finales}

La práctica variada de la Educación Física es atractiva. Algunos estudiantes reciben la clase de EF con una calidad excelente, pero otros tienen restricciones debido a la cultura, religión, economía y estatus social. Algunos nunca han recibido una clase de EF. La educación de calidad es una preocupación a nivel mundial (Hardman et al., 2013). Este es un hecho que ha sido señalado en todas las áreas del conocimiento y la Educación Física no es la excepción.

Las acciones para la EFC desde 1978 hasta el 2015 se inician con la Carta Fundamental para la Educación Física el Deporte (1978), en particular, se destaca que es un derecho humano y sus 11 artículos protegen la provisión de la Educación Física. Posteriormente, se modifica la Carta Fundamental y en el mismo año 2015 surgen las orientaciones para los que hacen las políticas acerca de la Educación Física de Calidad, que comprende: inclusión, aspectos de género, garantía de calidad, flexibilidad curricular, asociación con la comunidad, entrenamiento profesional y desarrollo de elementos de apoyo para la Educación
Física. Las orientaciones existen, ¿qué corresponde entonces ahora? Se sugiere:

- Lograr compromisos de las autoridades respectivas de cada país a cumplir de hecho y derecho (palabra y acción) estos acuerdos internacionales, y se hace necesario reflexionar acerca de los alcances en materia de actividad física, Educación Física y deporte en cada país.

- Es necesario el trabajo interministerial para el repunte de la actividad física, en particular, en los lugares en donde existen ministerios diversos para atender la Educación Física, el deporte, o en aquellos casos en donde hay ministerios o entes gubernamentales diferentes, para Educación Básica y otro para Educación Universitaria.

- Documentar los beneficios de la actividad física, de la creación de hábitos por medio de la Educación Física en el sistema educativo.

- Informar acerca de la actividad física a nivel local y nacional, por ejemplo, hacer campañas mediáticas.

- Investigar y publicar acerca de la actividad física, impulsarla desde instituciones de gobierno con una agenda nacional de investigación en la cual sea incluida la temática de la EFC y el tema de salud pública a través de una vida activa.

- Generar concursos que impulsen el trabajo en estas áreas.

- Proyectar el trabajo que se realiza en el país a nivel internacional, de esta manera se proyectan en la comunidad internacional los alcances que se van obteniendo.

A nivel regional (América Latina), hay organizaciones con las cuales se pueden establecer alianzas, por ejemplo: ALBA, MERCOSUR, CELAC, UNASUR, Consejo Latinoamericano de Ciencias Sociales (CLACSO) y trabajar en la agenda de la Educación Física de Calidad. También apoyarse en la Oficina Regional de la Educación para América Latina y el 
Caribe (OREALC) de la UNESCO, la Organización para la Cooperación y el Desarrollo Económico (OCDE) y el Centro Interamericano de Estudios e Investigaciones para el Planeamiento de la Educación/CINTERPLAN-OEA. En fin, hacer alianzas estratégicas que permitan el apoyo para profundizar acerca de lo que se está haciendo en la región para seguir impulsando la EFC y que ser partícipe de la construcción de equipos de trabajo permita al docente, pero también a las autoridades, pensar en ser parte de la constitución de una red global/glocal por la calidad educativa. Las políticas son importantes, pero por sí solas no van a lograr el cambio. Es necesario que todos impulsen los procesos y se apropien de la documentación existente para elevar el estatus de la EF en el sistema educativo. Latinoamérica también debe fortalecer sus organizaciones académicas regionales, de modo que permita el crecimiento en esta área, tal como lo observamos en los otros continentes.

\section{Referencias}

Amusa, L. O., \& Toriola, A. L. (2010). The changing phases of Physical education in Africa: Can a uniquely African model emerge? African Journal for Physical Activity and Health Sciences, 16(4), 666-680. https://doi.org/10.4314/ajpherd.v16i4.64095

Demirel, D. H., \& Yildiran, I. (2013). The Philosophy of Physical Education and Sport from Ancient Times to the Enlightenment. European Journal of Educational Research, 2(4), 191-202.

https://doi.org/10.12973/eu-jer.2.4.191

Díaz, G. B., Liermo, A. P., Puig, A. R., \& Savigne, M. B. (2013). Historia de la Educación Física en Cuba. Actividad Física y Ciencias (Revista electrónica), 5(1), 1-15. https://bit.ly/36dNfbL

Díaz, G. B., Morales Ferrer, A. M., \& Goslin, A. (2016). En R. López de D’Amico, T. Benn \& G. Pfister (Eds.). Women and Sport in Latin America. Routledge. https://doi.org/10.4324/9781315736020-9

Galakof, A. (2015, 21 julio). The Figure of the Gentleman in 19th century Victorian England: The
re-Fashioning of a Manhood Ideal. Buzz... littéraire. https://bit.ly/36dSw33

Goodbody, J. (1982). The illustrated history of gymnastics. Stanley Paul.

Hardman, K. (2004). An up-date on the status of physical education in schools worldwide: technical report for the World Health Organization. World Health Organization. https://bit.ly/2s0ELWv

Hardman, K. (2008). Physical education in schools: a global perspective. Kinesiology: International journal of fundamental and applied kinesiology, 4O(1), 5-28. https://bit.ly/36awjms

Hardman, K., \& Marshall, J. (2000). World-wide survey of the state and status of school physical education: The final report to the International Olympic Committee. University of Manchester.

https://doi.org/10.1177/1356336x000063001

Hardman, K., \& Marshall, J. (2009). Second World-wide Survey of School Physical Education. Final Report. ICSSPE.

Hardman, K., Murphy, C., Routen, A., \& Tones, S. (2013). UNESCO NWCPEA: Worldwide Survey of School Physical Education. Final Report. UNESCO. https://bit.ly/38gVW74

Ho, W. K. Y., Ahmed, D., Wong, B., Huang, F., López de D’Amico, R., Dinold, M., \& Branislav, A. (2016). Quality Physical Education and Global Concern - Ways Ahead and Future Development. Revista Electrónica Actividad Física y Ciencias, 8(1), 60-70. http://bit.ly/2PhJRqo

Ho, W. K. Y., López D’Amico, R., Dinold, M., Benn, T., Antala, B., Wong, B., Huang, F., \& Holzweg, M. (2014). Quality development and reform in physical education. En C. Scheuer, B. Antalay \& M. Holzweg (Eds.). Physical Education: Quality in Management and Teaching (pp.46-61). Logos.

https://bit.ly/2PpO8XI

Ho, W. K. Y., Ahmed, M. D., Keh, N. C., Khoo, S., Tan, C., Dehkordi, M. R., Gallardo, M., Lee, K., Yamaguchi, Y., Wang, J., Liu, M., \& Huang, F. 
(2017). Professionals' perception of quality physical education learning in selected Asian cities. Cogent Education, 4(1), 1-17.

https://doi.org/10.1080/2331186x.2017.1408945

Ho, W. K. Y., Ahmed, D., López de D’Amico, R., Ramos, A., Ferreira, E. L., Rocha Ferreira, M. B., Franco Amaral, S. C., Ceballos Gurrola, O., Béquer Díaz, G., Ramos, A., Hoyos, L. A., Jasmín, A., Rivera, A., Leon Van Niekerk, R., Huang, F., \& Wong, B. (2018). Measuring the perception of quality physical education in Latin American professionals. Revista Brasileira de Ciências do Esporte, 40(4), 361-369. https://doi.org/10.1016/j.rbce.2018.05.006

Ho, W. K. Y. (2018). The Study of Quality Physical Education - Past, Recent Researches and Attempts for Quality Improvement. Lecture series prepared for the $60^{\text {th }}$ Anniversary of Tianjin University of Sport (June 2018). https://bit.ly/33YaEwj

Ceballos, O., Jáuregui, A., Salinas, A., Ávila, A., Villafuerte, A., Martínez, C., Medina, C., Salazar, C. (2018). Hacia una estrategia nacional para la prestación de Educación Física de calidad en el nivel básico del Sistema Educativo Mexicano. Instituto Nacional de Salud Pública; CONADE; UNESCO; OPS; OMS. https://bit.ly/2YlyK2T

López de D’Amico, R. (2013). Pedagogies for teaching PE in the 21st Century. The Global Journal of Health and Physical Education Pedagogy 2(4), 32-43. https://bit.ly/2Rs8zpz

López de D’Amico, R., Ho, W., Antala, B., Dinold, M., Benn, T., \& Holzweg, M. (2014c). Voces Acerca de la Educación Física en América Latina. Revista Des-encuentros, 11, 6-14. http://bit.ly/34jMwUQ

López de D’Amico, R., Ho, W. K. Y., Benn, T., Dinold, M., \& Branislav, A. (2014a). Gestión e investigación en organizaciones académicas de educación física y deporte. En E. Ferreira (Ed.). Focuses on Physical Education (pp.91-142). NGIME-UFJF.

López de D’Amico, R., Ramos, A., \& Guerrero, G. (2014b). Physical education and health in the Venezuelan social transformation context. In M. K. Chin
\& C. R. Edginton (Eds.). Physical Education and Health. Global Perspectives and Best Practice (pp.545558). SAGAMORE.

https://bit.ly/2r9AdND

López de D’Amico, R. L., Ho, W. K. Y., Antala, B., Benn, T., Dinold, M., \& Holzweg, M. (2018). Compartiendo perspectivas globales acerca de la Educación Física de calidad. Educere, 22(72), 325341. https://bit.ly/2LvPSxx

López de D’Amico, R., \& Guerrero de Hojas, G. (2018). Transformación curricular y pedagógica en Educación Media en Venezuela: caso Educación Física. The Journal of the Latin American Socio-cultural Studies of Sport (ALESDE), 9(2), 119-133.

https://bit.ly/2OUIaPK

López-Taylor, J., Jáuregui-Ulloa, E., \& González-Villalobos, M. (2014). Physical Education in Mexico: Experiences and trends related to Physical Activity and Health. In M. K. Chin \& C. R. Edginton (Eds.). Physical Education and Health Global Perspectives and Best Practice (pp.311-324). SAGAMORE. https://bit.ly/2r9AdND

Le Masurier, G., \& Corbin, C. B. (2006). Top 10 reasons for Quality Physical Education. Journal of Physical Education, Recreation \& Dance, 77(6), 44-53. https://doi.org/10.1080/07303084.2006.10597894

NASPE (2004). Moving Into The Future: National Standards for Physical Education. McGraw-Hill Education. https://bit.ly/2RpeSu3

NASPE (2007). What constitutes a highly qualified physical education teacher. AAHPERD.

https://bit.ly/38i3bvx

Ramírez Torrealba, J. (2009). Fundamentos teóricos de la recreación, la educación física y el deporte: una introducción hacia la físico-corporalidad. Episteme. https://bit.ly/2qsJ6Bv

UNESCO (2004). Education for all: the quality imperative; EFA global monitoring report, 2005. UNESCO. http://bit.ly/2RLUDXV

UNESCO (2005). Rapport final: Séminaire UNES- 
CO sur la qualité de l'éducation physique et sportive. UNESDOC Biblioteca Digital.

https://bit.ly/34YEuSH

UNESCO/MINEPS V. (2013). Declaración de Berlin. UNESDOC. Biblioteca Digital.

http://bit.ly/35jEVHm

UNESCO / MINEPS V. (2013). Declaración de Berlín. Disponible en: https://bit.ly/33Y53Gj

UNESCO (2015a). Educación Física de CalidadGuía para los responsables politicos. UNESCO. https://bit.ly/2RqNGv3
UNESCO (2015b). Educación Física de Calidad. Guía para los representantes politicos. Metodología. UNESDOC Biblioteca Digital.

http://bit.ly/2PihiJB

UNESCO (2015c). Políticas de Educación Física de Calidad. UNESDOC Biblioteca Digital. http://bit.ly/2LT8V50

UNESCO (2017). Quality Physical Education (QPE) Policy Project. UNESCO. http://bit.ly/34jL6K9

\section{CÓMO CITAR:}

López-D’Amico, R. (2019). Educación física de calidad: ¿De dónde surge este planteamiento? Revista Caribeña de Investigación Educativa (RECIE), 3(2), 33-45. https://doi.org/10.32541/recie.2019.v3i2.pp33-45 